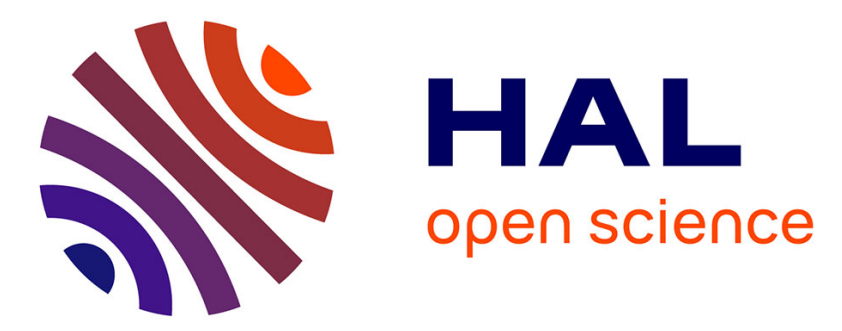

\title{
Adaptive Distributed Parameter and Input Estimation in Plasma Tokamak Heat Transport
}

\author{
Sarah Mechhoud, Emmanuel Witrant, Luc Dugard, Didier Moreau
}

\section{To cite this version:}

Sarah Mechhoud, Emmanuel Witrant, Luc Dugard, Didier Moreau. Adaptive Distributed Parameter and Input Estimation in Plasma Tokamak Heat Transport. CPDE 2013 - 1st IFAC Workshop on

Control of Systems Modeled by Partial Differential Equations, Sep 2013, Paris, France. hal-00931642

\section{HAL Id: hal-00931642 \\ https://hal.science/hal-00931642}

Submitted on 15 Jan 2014

HAL is a multi-disciplinary open access archive for the deposit and dissemination of scientific research documents, whether they are published or not. The documents may come from teaching and research institutions in France or abroad, or from public or private research centers.
L'archive ouverte pluridisciplinaire HAL, est destinée au dépôt et à la diffusion de documents scientifiques de niveau recherche, publiés ou non, émanant des établissements d'enseignement et de recherche français ou étrangers, des laboratoires publics ou privés. 


\title{
Adaptive Distributed Parameter and Input Estimation in Plasma Tokamak Heat Transport
}

\author{
Sarah MECHHOUD* Emmanuel WITRANT * \\ Luc DUGARD* ${ }^{*}$ Didier MOREAU ${ }^{* *}$ \\ * UJF-Grenoble 1/CNRS, Grenoble Image Parole Signal Automatique \\ (GIPSA-lab), UMR 5216, B.P. 46, F-38402 St Martin d'Hères, France \\ ** CEA, IRFM, F-13108 Saint Paul-Lez-Durance, France \\ E-mail: $\{$ sarah.mechhoud, emmanuel.witrant, luc.dugard $\}$ \\ @gipsa-lab.grenoble-inp.fr, didier.moreau@cea.fr
}

\begin{abstract}
In this paper, the adaptive estimation of spatially varying diffusion and source term coefficients for a linear parabolic partial differential equation describing tokamak plasma heat transport is considered. An estimator is defined in the infinite-dimensional framework having the system state and the parameters' estimate as its states. Our scheme allows to estimate constant, spatially distributed and spatio-temporally distributed parameters as well as input with known upper bounds in time. While the parameters convergence depends on the plant signal richness assumption, the state convergence is established using the Lyapunov approach. Since the estimator is infinite-dimensional, the Galerkin finite-dimensional technique is used to implement it. In silico simulations are provided to illustrate the performance of the proposed approach.
\end{abstract}

Keywords: Thermonuclear fusion, distributed parameter systems, input state and parameter estimation, adaptive infinite-dimensional estimation, Galerkin method

\section{INTRODUCTION}

In a controlled thermonuclear fusion reactor, the plasma thermal diffusivity and heating energy play an important role in the development of new challenging control strategies. These parameters describe the plasma heat transport, which affects the current and pressure profiles and therefore the plasma confinement itself. Presently, to the best of our knowledge, there are no physical laws describing these variables. In fact, for the diffusion coefficient, only some empirical models exist (Hoang et al. (1998)). Each one depends on various conditions (tokamak dimensions, discharge parameters, temperature profile, etc.). The source term (the heating energy absorbed by the particles), due to its dependence on the plasma-wave coupling is also difficult to model based on theoretical knowledge.

The plasma heat transport in tokamak is described by a linear parabolic partial differential equation (PDE) with reaction and diffusion coefficients. While the reaction parameter is assumed to be constant (given by Hoang et al. (1998) empirical model), the diffusion coefficient and source term are distributed space-time parameters. Thus, the plasma heat transport can be considered as a

* This work was carried out within the framework of the European Fusion Development Agreement and the French Research Federation for Fusion Studies. It is supported by the European Communities under the contract of Association between EURATOM and CEA. The views and opinions expressed herein do not necessarily reflect those of the European Commission.

This project receives partial funding provided by the Bonus Quality Research of Grenoble-INP. dynamical distributed parameter system (DPS).

In our previous works (Mechhoud et al. (2013a), Mechhoud et al. (2013b)), we attempted to solve this problem in a finite-dimensional framework using Galerkin formulation and a modified Kalman filter. In order to investigate the possible improvements brought by considering the distributed dynamics directly in the estimation approach, this paper is focused on the infinite-dimensional framework using on-line or adaptive estimation techniques.

Off-line methods for functional parameters in DPS were extensively studied both in finite and infinite-dimensional frameworks (Banks and Kunisch (1989), Yu and Seinfeld (1988), etc.). The identification methods were based mainly on output least squares and maximum likelihood estimators. These techniques lead generally to nonlinear optimization problems. An on-line estimator utilizes the PDE characteristics and consequently the problem remains linear.

Adaptive estimation for infinite dimensional dynamic systems with both constant and spatially varying parameters with known inputs was first addressed in Baumeister et al. (1997) and their earlier works (for example: Dr. Scondo and Dr. Demetriou's Ph. D theses). They also established the abstract framework of this problem using functional analysis tools and Sobolev spaces properties. In Orlov and Bentsman (2000), constructively enforceable identifiability conditions based on manipulable quantities were introduced for the first time. Unlike Baumeister et al. (1997), the proposed adaptive estimator used the PDE features to reduce the order of the spatial derivatives by obviating the 
repetition of the spatial derivative structure of the plant in the tuning laws.

Two main contributions are presented in this work. First, the identifiability conditions of the simultaneous diffusion/input estimation problem are shown. Since the input is unknown, these conditions are passive. Then, only constant or spatially varying input/parameters' simultaneous on-line estimation is considered. This part can be regarded as an extension of Orlov and Bentsman (2000) work to the joint input/parameter identification problem. In the second part, distributed slowly time-varying input/parameter are examined. We demonstrate that a region of convergence of the state error can be expressed explicitly as a function of the identifiers' tuning parameters. After that, inspired by Wada et al. (2006) paper, adaptive estimation of distributed time-varying input/parameter with known upper bounds is discussed.

The question of input estimation is not only related to plasma heat transport but arises more generally in fault detection and inverse problems.

This paper is organized as follows. The electron heat transport model and the framework of our PDE problem are presented in Section II. In Section III, we treat the diffusion and source term identifiability conditions. The adaptive estimators for functional (spatially varying) and distributed time-varying state, input and parameters are considered in Section IV. In order to illustrate the performance of the proposed identifiers, computer simulations are carried out in Section V.

\section{ELECTRON HEAT TRANSPORT MODEL}

Assuming the poloidal and toroidal axisymmetry, the tokamak may be considered as an infinite cylinder where space variations occur only along the radius $a$. Applying the energy conservation principle, the electron heat transport model is given by the following parabolic partial differential equation (Hinton and Hazeltine (1976)):

$\frac{\partial(n T)}{\partial t}=\frac{2}{3} \frac{1}{r} \frac{\partial}{\partial r}\left(r n \bar{\chi}_{e}(r, t) \frac{\partial T}{\partial r}\right)-\frac{1}{\tau} n T(r, t)+\frac{2}{3} P_{e}(r, t)$, where $t$ is the time, $r$ is the radial variable along $a, \bar{\chi}_{e}$ is the electron diffusivity, $\tau(<\infty)$ is a damping time modelling energy losses, $T$ is the electron temperature, $n$ is the electron density and $P_{e}$ is the power density absorbed by the particles from an external heating system.

The spatio-temporal variations of the electron density are assumed to be negligible with respect to the temperature variations during the heating process, and using the normalized variable $x=\frac{r}{a},(1)$ can be written as:

$$
\left\{\begin{array}{l}
\frac{\partial T}{\partial t}=\frac{1}{x} \frac{\partial}{\partial x}\left(x \chi_{e}(x, t) \frac{\partial T}{\partial x}\right)-\frac{1}{\tau} T(x, t)+S(x, t) \\
\frac{\partial T}{\partial x}(0, t)=0 ; T(1, t)=0 \\
\left.T(x, 0)=0 ; \quad x \in \Omega ; \quad t \in] 0, t_{f}\right]
\end{array}\right.
$$

where $\Omega$ is the interval ]0,1[. The normalized source term and diffusivity coefficient are respectively given by:

$$
\left\{\begin{array}{l}
S(x, t)=\frac{2}{3 n} P_{e}(x, t) \\
\chi_{e}(x, t)=\frac{2}{3 a^{2}} \overline{\chi_{e}}(r, t)
\end{array}\right.
$$

In system (2), the second and third equations represent initial and boundary conditions, chosen to guaranty the symmetry and boundedness of the solution near zero. The value 0 refers to an equilibrium temperature level and not to the absolute zero temperature. The diffusion coefficient $\chi_{e}$ is assumed to belong to the space $Q_{\chi_{2}}=\{f: f(x, t) \in$ $\left.C^{0}\left(0, t_{f} ; C^{1}(\Omega)\right), f(x, t)>c>0\right\}$, the reaction coefficient $\tau$ in $\mathbb{R}_{*}^{+}$and the source term $S$ in $Q_{S}:=C^{0}\left(0, t_{f} ; C^{0}(\Omega)\right)$. These assumptions guarantee the existence, uniqueness and differentiability of the classical solution of PDE (2) in $Q_{T}:=C^{1}\left(0, t_{f} ; C^{2}(\Omega)\right)($ see Evans $(2010)$, chapter 07 , page 375 ).

In the following, $\tau$ is considered as a known variable given by Hoang et al. (1998) experimental model.

\section{DIFFUSION AND SOURCE TERM IDENTIFIABILITY}

The input/parameter estimation convergence is guaranteed if we can ensure the problem identifiability: whether it is possible to uniquely extract the solution for the model unknown variables from the measurements.

In the finite-dimensional framework, this problem has been extensively studied (see Raue et al. (2010) and references therein) both for structural (noise-free model) and practical aspects. In adaptive estimation techniques, this is equivalent to ensure plant signals richness. The finitedimensional notion of persistence of excitation was extended to infinite-dimensional systems first by Baumeister et al. (1997) and Demetriou and Rosen (1997). Later, in Orlov and Bentsman (2000), constructively enforceable identifiability conditions were given and the construction of persistently exciting inputs was shown. In this paper, the structural identifiability question is treated in the spirit of Orlov and Bentsman (2000) paper, extended to (1) deal with both diffusion/source term reconstruction and the only available inputs under which identifiability is considered are the PDE's (2) boundary conditions.

Definition: (Orlov and Bentsman (2000)) A set of parameters $\left\{\chi_{e}(x, t), S(x, t)\right\}$ of the PDE (2) is said to be identifiable with the corresponding boundary conditions if and only if:

$$
\begin{aligned}
& \forall x \in] 0,1\left[, \forall t \geq 0, \forall \bar{\chi}_{e} \neq \chi_{e}, \forall \bar{S} \neq S:\right. \\
& \operatorname{div}\left(\Delta \chi_{e} \frac{\partial T}{\partial x}\right)+\Delta S=0 \Rightarrow \Delta \chi_{e}=\Delta S=0,
\end{aligned}
$$

where div is the divergence operator in the cylindrical coordinates supposing a gradient in the $x$ direction only: $\operatorname{div}():.=\frac{1}{x} \frac{\partial}{\partial x}(x \cdot), \Delta \chi_{e}=\chi_{e}-\bar{\chi}_{e}$ and $\Delta S=S-\bar{S}$.

The concept of persistent excitation relies on the ability of finding the Fourier expansion of the plant state on an arbitrary orthonormal basis in $L_{2}(0,1)$. In this, $T(x, t)$ can be written as: 


$$
T(x, t)=\sum_{n=0}^{\infty} l_{n}(t) \cos (\pi n x) .
$$

where the Fourier coefficients $l_{n}$ are linearly independent functions (for more details see Orlov and Bentsman (2000)). Sufficient conditions for simultaneous diffusion and source term identification are given as follows.

Theorem 1. If the boundary conditions (and the input) of the PDE (2) generate a persistent excitation of the system (such that the Fourier coefficients of the plant state are linearly independent), then the parameter $\chi_{e}$ and the external input $S$ are identifiable (with these boundary conditions).

Proof. Substituting the Fourier expansion of $T$ given in (5) into (4) yields:

$$
\begin{aligned}
& \sum_{n=0}^{\infty}\left\{\left[\operatorname{div}\left(\Delta \chi_{e}\right) n \pi l_{n}(t)+\frac{n \pi}{x} \Delta \chi_{e} l_{n}(t)\right] \sin (n \pi x)+\right. \\
& \left.\quad+(n \pi)^{2} \Delta \chi_{e} l_{n}(t) \cos (n \pi x)\right\}-\Delta S=0 .
\end{aligned}
$$

The input $S$ can also be written as a Fourier series:

$$
S(x, t)=\sum_{n=0}^{\infty} \omega_{n}(t) \cos (n \pi x)
$$

where $\left\{\omega_{n}(t)\right\}_{n=0}^{n=\infty}$ are linearly independent. Substituting $S$ in (6) gives:

$$
\begin{aligned}
& \sum_{n=0}^{\infty}\left\{\left[\operatorname{div}\left(\Delta \chi_{e}\right) n \pi l_{n}(t)+\frac{n \pi}{x} \Delta \chi_{e} l_{n}(t)\right] \sin (n \pi x)+\right. \\
& \left.+\left[(n \pi)^{2} \Delta \chi_{e} l_{n}(t)-\Delta \omega_{n}(t)\right] \cos (n \pi x)\right\}=0 .
\end{aligned}
$$

Since the sets $\{\cos (n \pi x)\}$ and $\{\sin (n \pi x)\}$ have no intersecting zero and $\left\{l_{n}(t)\right\}_{n=0}^{\infty}$ are linearly independent (persistent excitations hypothesis), we conclude that (7) implies:

$$
\Delta \chi_{e}=0 \text { and }\left(\Delta \omega_{n}=0 \Leftrightarrow \Delta S=0\right) .
$$

Remark: Since the input is unknown and the aim of this work is to estimate it simultaneously with the diffusion coefficient, unlike what was done in Orlov and Bentsman (2000), input persistent generators cannot be constructed. The persistent excitation of the plant is investigated $a$ posteriori, once the measurements are available by checking the linear dependence of the plant Fourier coefficients unless a simulator on which tests can be carried out is accessible.

\section{ADAPTIVE ESTIMATOR DESIGN FOR DISTRIBUTED TIME-SPACE INPUT AND DIFFUSION COEFFICIENT}

For sufficiently rich signals, the simultaneous estimation of the input and the diffusion coefficient can be achieved using an adaptive estimator. The adaptive law is developed based on stability considerations or using simple optimization techniques to minimize the output error equation. In this section, we first assume that the parameters $(S$ and $\chi_{e}$ ) are time independent. Our initial objective is to demonstrate that under this assumption, a stable adaptive estimator for the simultaneous problem exists and guarantees the $L_{2}$ and point-wise convergence of both state and parameters (input and diffusion coefficient). The problem of distributed slowly time varying coefficients and time-varying parameters with known upper bounds will be addressed in the next subsection.

\subsection{Adaptive estimator for spatially varying parameters}

The adaptive identifier is a model-based estimator. It takes the form of PDE (2) to which an innovation term (correction) is added and a gradient-type update law for the parameters' estimate is associated. Supposing that distributed sensors are available and measure the system state, this estimator is described by:

$$
\begin{aligned}
& \left\{\begin{aligned}
\frac{\partial \hat{T}}{\partial t}= & \frac{1}{x} \frac{\partial}{\partial x}\left(x \hat{\chi_{e}}(x, t) \frac{\partial \hat{T}}{\partial x}\right)-\frac{1}{\tau} \hat{T}(x, t)+\hat{S}(x, t) \\
& -\vartheta_{0}(\hat{T}(x, t)-T(x, t))
\end{aligned}\right. \\
& \frac{\partial \hat{T}}{\partial x}(0, t)=\hat{T}(1, t)=0 ; \quad \hat{T}(x, 0)=\hat{T}_{0}(x) \geq 0 ; \\
& \left\{\begin{array}{l}
\frac{\partial \hat{\chi}_{e}}{\partial t}=\vartheta_{1} \frac{\partial}{\partial x}(\hat{T}-T) \frac{\partial \hat{T}}{\partial x} ; \hat{\chi}_{e}(x, 0)=\hat{\chi}_{e_{0}}(x) ; \\
\frac{\partial \hat{S}}{\partial t}=-\vartheta_{2}(\hat{T}-T) ; \hat{S}(x, 0)=\hat{S}_{0}(x) ; \\
\forall x \in] 0,1[; t \geq 0,
\end{array}\right.
\end{aligned}
$$

where $\vartheta_{i} \geq 0, i=0,1,2$ are the adaptation gains, $\hat{\chi}_{e_{0}}(x)>0$ is a smooth function and $S_{0}(x)$ is a continuous function.

Theorem 2. If the plant (2) is identifiable (under 1' conditions), the adaptive identification law given by (8) combined with the parameters identifiers in (9) ensure the $L_{2}$ convergence of the state and parameters deviations.

Proof. Let us first define the state and parameters deviations

$$
\left\{\begin{array}{l}
\Delta T=\hat{T}-T, \frac{\partial \Delta T}{\partial x}(0, t)=\Delta T(1, t)=0 \\
\Delta \chi_{e}(x, t)=\hat{\chi_{e}}(x, t)-\chi_{e}(x) \\
\Delta S(x, t)=\hat{S}(x, t)-S(x)
\end{array}\right.
$$

and their derivatives:

$$
\left\{\begin{aligned}
\frac{\partial \Delta T}{\partial t} & =\operatorname{div}\left(\chi_{e}(x) \frac{\partial \Delta T}{\partial x}\right)+\operatorname{div}\left(\Delta \chi_{e}(x, t) \frac{\partial \hat{T}}{\partial x}\right) \\
& -\left(\frac{1}{\tau}+\vartheta_{0}\right) \Delta T(x, t)+\Delta S \\
\frac{\partial \Delta \chi_{e}}{\partial t} & =\vartheta_{1} \frac{\partial \hat{T}}{\partial x} \frac{\partial \Delta T}{\partial x} \\
\frac{\partial \Delta S}{\partial t} & =-\vartheta_{2} \Delta T(x, t)
\end{aligned}\right.
$$

Since $\hat{\chi}_{e_{0}}(x)>0$ and $\tau \in \mathbb{R}_{*}^{+}$are bounded, $\hat{S}(x)$ is a continuous bounded function. There exists a unique 
local solution for the global system (2), (8) and (9).Thus the problem is well-posed and we introduce the following Lyapunov functional

$$
\begin{gathered}
V(t)=\frac{1}{2} \int_{0}^{1}\left([\Delta T(x, t)]^{2}+\frac{1}{\vartheta_{1}}\left[\Delta \chi_{e}(x, t)\right]^{2}\right. \\
\left.+\frac{1}{\vartheta_{2}}[\Delta S(x, t)]^{2}\right) d x .
\end{gathered}
$$

Taking into account the system (11) and using the Gauss divergence formula:

$$
\int_{0}^{1} d i v\left(\chi_{e} \frac{\partial \Delta T}{\partial x} \Delta T\right) d x=\left.\chi_{e} \frac{\partial \Delta T}{\partial x} \Delta T\right|_{0} ^{1}
$$

and:

$$
\operatorname{div}\left(\chi_{e} \frac{\partial \Delta T}{\partial x} \Delta T\right)=\Delta T \operatorname{div}\left(\chi_{e} \frac{\partial \Delta T}{\partial x}\right)+\chi_{e}\left(\frac{\partial \Delta T}{\partial x}\right)^{2}
$$

we obtain the following integration by parts for the divergence term:

$\int_{0}^{1} \operatorname{div}\left(\chi_{e} \frac{\partial \Delta T}{\partial x}\right) \Delta T d x=\left.\chi_{e} \frac{\partial \Delta T}{\partial x} \Delta T\right|_{0} ^{1}-\int_{0}^{1} \chi_{e}\left(\frac{\partial \Delta T}{\partial x}\right)^{2} d x$.

The time derivative of the Lyapunov functional (12) is given by:

$$
\begin{aligned}
& \dot{V}(t)=\left.\chi_{e}(x, t) \frac{\partial \Delta T}{\partial x} \Delta T(x, t)\right|_{0} ^{1}-\int_{0}^{1} \chi_{e}(x, t)\left(\frac{\partial \Delta T}{\partial x}\right)^{2} d x \\
& +\left.\Delta \chi_{e}(x, t) \frac{\partial \hat{T}}{\partial x} \Delta T(x, t)\right|_{0} ^{1}-\int_{0}^{1} \Delta \chi_{e}(x, t) \frac{\partial \Delta T}{\partial x} \frac{\partial \hat{T}}{\partial x} d x \\
& -\int_{0}^{1}\left(\frac{1}{\tau}+\vartheta_{0}\right)[\Delta T(x, t)]^{2} d x+\int_{0}^{1} \Delta S(x, t) \Delta T(x, t) d x \\
& +\int_{0}^{1} \Delta \chi_{e}(x, t) \frac{\partial \hat{T}}{\partial x} \frac{\partial \Delta T}{\partial x} d x-\int_{0}^{1} \Delta S(x, t) \Delta T(x, t) d x \\
& =-\int_{0}^{1} \chi_{e}(x, t)\left(\frac{\partial \Delta T}{\partial x}\right)^{2} d x-\int_{0}^{1}\left(\frac{1}{\tau}+\vartheta_{0}\right)[\Delta T(x, t)]^{2} d x \\
& \leq-\int_{0}^{1}\left(\frac{1}{\tau}+\vartheta_{0}\right)[\Delta T(x, t)]^{2} d x \leq 0
\end{aligned}
$$

This proves the boundedness of Lyapunov functional (12) for all $t \geq 0$ and $L_{2}$ boundedness of system solutions (8),(9). In this special case, the invariance principle can be used (see Orlov and Bentsman (2000) and references therein). Therefore, the trajectories of system (8),(9) converge to the maximal invariant subset of a set of solutions of (8),(9), for which $\dot{V}=0$. This implies $\Delta T=0$ and leads to the following expression:

$$
\left.\operatorname{div}\left(\Delta \chi_{e}(x, t) \frac{\partial T}{\partial x}\right)+\Delta S(x, t)=0 ; \forall x \in\right] 0,1[, t \geq 0(
$$

With the identifiability hypothesis, it follows that:

$$
\left.\Delta \chi_{e}(x, t)=\Delta S(x, t)=0 ; \forall x \in\right] 0,1[, t \geq 0 .
$$

and thus, we deduce that

$$
\lim _{t \rightarrow+\infty} \int_{0}^{1}\left\{(\Delta T)^{2}+(\Delta S)^{2}+\left(\Delta \chi_{e}\right)^{2}\right\} d x=0 .
$$

Finally, using the same methodology as the one proposed in Orlov and Bentsman (2000), parameters' point-wise convergence can be asserted.

\subsection{Case of the distributed slowly time-varying parameters}

When dealing with slowly time varying parameters, $L_{2}$ convergence for all $\Delta T \in Q_{T}$ can no longer be guaranteed: only a region of convergence can be established.

In the infinite-dimensional framework, parameters' slowly time varying and boundedness assumptions can be given by:

$$
\begin{array}{r}
\forall x \in] 0,1\left[, \forall t \geq 0, \exists \epsilon_{1}, \epsilon_{2}, \epsilon_{1}^{\star}, \epsilon_{2}^{\star}:\right. \\
\left\{\begin{array}{l}
\left\|\frac{\partial \chi_{e}}{\partial t}(., t)\right\|_{2} \leq \epsilon_{1} ; \quad\left\|\frac{\partial S}{\partial t}(., t)\right\|_{2} \leq \epsilon_{2} \\
\left\|\Delta \chi_{e}(., t)\right\|_{2} \leq \epsilon_{1}^{\star} ; \quad\|\Delta S(., t)\|_{2} \leq \epsilon_{2}^{\star}
\end{array}\right.
\end{array}
$$

Lemma 3. Under assumptions (14), parameters' update laws given in (8), (9) guarantee the $L_{2}$ convergence of the state error in the region:

$$
\Pi=\left\{\Delta T \in Q_{T}:\|\Delta T(., t)\|_{2} \leq \sqrt{\frac{\epsilon_{1} \epsilon_{1}^{\star} \vartheta_{2}+\epsilon_{2} \epsilon_{2}^{\star} \vartheta_{1}}{\vartheta_{0} \vartheta_{1} \vartheta_{2}}}, \forall t \geq 0\right\}
$$

Proof. The proof is based on Theorem 2. As we consider space-time varying parameters $\left(\chi_{e}(x, t)\right.$ and $\left.S(x, t)\right)$, the derivative of Lyapunov functional (12) becomes:

$$
\begin{aligned}
\dot{V}(t)= & -\int_{0}^{1} \chi_{e}(x, t)\left(\frac{\partial \Delta T}{\partial x}\right)^{2} d x+\frac{1}{\vartheta_{1}} \int_{0}^{1} \frac{\partial \chi_{e}}{\partial t} \Delta \chi_{e}(x, t) d x \\
& -\int_{0}^{1}\left(\frac{1}{\tau}+\vartheta_{0}\right)[\Delta T(x, t)]^{2} d x+\frac{1}{\vartheta_{2}} \int_{0}^{1} \frac{\partial S}{\partial t} \Delta S(x, t) d x
\end{aligned}
$$

Using the Cauchy-Schwarz inequality and assumptions (14)

$$
\dot{V}(t) \leq-\int_{0}^{1} \vartheta_{0}[\Delta T(x, t)]^{2} d x+\frac{\epsilon_{1} \epsilon_{1}^{\star}}{\vartheta_{1}}+\frac{\epsilon_{2} \epsilon_{2}^{\star}}{\vartheta_{2}} .
$$

where $1 / \tau$ has been implicitly included in the design parameter $\vartheta_{0}$. Thus,

$$
\left(\|\Delta T(x, t)\|_{2}^{2} \geq \frac{\epsilon_{1} \epsilon_{1}^{\star} \vartheta_{2}+\epsilon_{2} \epsilon_{2}^{\star} \vartheta_{1}}{\vartheta_{0} \vartheta_{1} \vartheta_{2}}\right) \Rightarrow(\dot{V} \leq 0) .
$$

Hence for $\Delta T \in Q_{T}-\Pi$, state and parameters errors are $L_{2}$ bounded. By choosing large tuning parameters $\vartheta_{i}, i=$ $0,1,2$, we can make the state error region $\Pi$ arbitrarily small. On the other hand, large tuning parameters may lead to noise amplification.

4.3 Adaptive estimation of space-time parameters with known upper bounds

Now, we assume that the parameters satisfy the following inequalities $\forall x \in] 0,1[, t \geq 0$ :

$$
\left\{\begin{array}{l}
\left\|\chi_{e}(x, t)-\chi_{e}(x, 0)\right\|_{2}^{2} \leq \xi_{0}, \\
\|S(x, t)-S(x, 0)\|_{2}^{2} \leq \gamma_{0},
\end{array}\right.
$$

where $\xi_{0}$ and $\gamma_{0}$ are known upper bounds and parameters $\chi_{e}$ and $S$ can be written as

$$
\left\{\begin{array}{l}
\chi_{e}(x, t)=\chi_{e}(x, 0)+\xi(x, t), \\
S(x, t)=S(x, 0)+\gamma(x, t) .
\end{array}\right.
$$


For the parameters' tuning laws (9), additional update laws on the new variables $\xi$ and $\gamma$, that characterize respectively $\chi_{e}$ and $S$ time variations, are selected as:

$$
\left\{\begin{array}{l}
\frac{\partial \hat{\xi}}{\partial t}=\left(\frac{\partial \Delta T}{\partial x}\right)^{2}\left\|\frac{\partial \hat{T}}{\partial x}(., t)\right\|_{2}^{2} \\
\frac{\partial \hat{\gamma}}{\partial t}=[\Delta T(x, t)]^{2} .
\end{array}\right.
$$

The state adaptation law is given by:

$$
\left\{\begin{aligned}
& \frac{\partial \hat{T}}{\partial t}=\operatorname{div}\left(\hat{\chi_{e}} \frac{\partial \hat{T}}{\partial x}\right)-\frac{1}{\tau} \hat{T}(x, t)+\hat{S}(x, t)-\vartheta_{0} \Delta T(x, t) \\
& \quad-\hat{\gamma}(x, t) \Delta T(x, t)+\operatorname{div}\left(\hat{\xi}\left\|\frac{\partial \hat{T}}{\partial x}\right\|_{2}^{2} \frac{\partial \Delta T}{\partial x}\right) \\
& \frac{\partial \hat{T}}{\partial x}(0, t)=\hat{T}(1, t)=0 ; \quad \hat{T}(x, 0)=\hat{T}_{0} \\
& \forall x \in[0,1], \quad \forall t \geq 0
\end{aligned}\right.
$$

Lemma 4. The state and parameters adaptive estimators (18), (9) and (17) guarantee the stability of the origin of the state and parameters errors as follows:

$$
\begin{gathered}
\lim _{t \rightarrow+\infty}\|\Delta T(x, t)\|_{2}=0 \\
\lim _{t \rightarrow+\infty} \Delta T(x, t)=\lim _{t \rightarrow+\infty} \Delta \chi_{e}(x, t)=\lim _{t \rightarrow+\infty} \Delta S(x, t)=0
\end{gathered}
$$

Proof. Introduce the following errors: $\forall x \in] 0,1[, \quad t \geq 0$ :

$$
\left\{\begin{array}{l}
\tilde{\gamma}(x, t)=\hat{\gamma}(x, t)-\gamma_{0}, \quad \tilde{S}(x, t)=\hat{S}(x, t)-S(x, 0), \\
\tilde{\xi}(x, t)=\hat{\xi}(x, t)-\xi_{0}, \quad \tilde{\chi}_{e}(x, t)=\hat{\chi}_{e}(x, t)-\chi_{e}(x, 0),
\end{array}\right.
$$

and define a new Lyapunov functional:

$$
V(t)=\frac{1}{2} \int_{0}^{1}\left\{(\Delta T)^{2}+\frac{1}{\vartheta_{1}} \tilde{\chi}_{e}^{2}+\frac{1}{\vartheta_{2}} \tilde{S}^{2}+\tilde{\gamma}^{2}+\tilde{\xi}^{2}\right\} d x
$$

where the space-time dependence is dropped for the sake of simplicity. The time derivative of $\Delta T$ becomes:

$$
\left\{\begin{array}{l}
\frac{\partial \Delta T}{\partial t}=\operatorname{div}\left(\chi_{e} \frac{\partial \Delta T}{\partial t}\right)+\operatorname{div}\left(\Delta \chi_{e} \frac{\partial \hat{T}}{\partial x}\right)-\hat{\gamma} \Delta T \\
\quad+\operatorname{div}\left(\hat{\xi}\left\|\frac{\partial \hat{T}}{\partial x}\right\|_{2}^{2} \frac{\partial \Delta T}{\partial x}\right)-\left(\frac{1}{\tau}+\vartheta_{0}\right) \Delta T+\Delta S \\
\frac{\partial \Delta T}{\partial x}(0, t)=\Delta T(1, t)=0 \\
\Delta T(x, 0)=0
\end{array}\right.
$$

Using integration by parts and divergence Gauss formula, $\dot{V}$ is given by

$$
\begin{aligned}
\dot{V}= & -\int_{0}^{1} \chi_{e}\left(\frac{\partial \Delta T}{\partial x}\right)^{2} d x+\int_{0}^{1} \xi \frac{\partial \hat{T}}{\partial x} \frac{\partial \Delta T}{\partial x} d x \\
& -\int_{0}^{1} \xi_{0}\left(\frac{\partial \Delta T}{\partial x}\right)^{2}\left\|\frac{\partial \hat{T}}{\partial x}\right\|_{2}^{2} d x-\int_{0}^{1} \gamma_{0}(\Delta T)^{2} d x(22 \\
& -\int_{0}^{1} \gamma \Delta T d x-\int_{0}^{1}\left(\frac{1}{\tau}+\vartheta_{0}\right)(\Delta T)^{2} d x
\end{aligned}
$$

From Cauchy-Schwarz inequality and assumptions (14) we get

$$
\begin{aligned}
\dot{V} \leq & -\vartheta_{0} \int_{0}^{1}(\Delta T)^{2} d x-\left(\gamma_{0}-\|\gamma\|_{2}^{2}\right)\|\Delta T\|_{2}^{2}\|\hat{T}\|_{2}^{2} \\
& -\left(\xi_{0}-\|\xi\|_{2}^{2}\right)\left\|\frac{\partial \Delta T}{\partial x}\right\|_{2}^{2}\left\|\frac{\partial \hat{T}}{\partial x}\right\|_{2}^{2} \\
& \leq-\vartheta_{0}\|\Delta T\|_{2}^{2} \leq 0 .
\end{aligned}
$$

As we are considering space-time varying parameters, the invariance principle can not be used. Inequality (23) implies that $\Delta T(., t) \in L_{2} \cap L_{\infty}, \tilde{\chi}_{e}(., t) \in L_{\infty}, \tilde{S}(., t) \in L_{\infty}$ and hence $\dot{\Delta T}(., t) \in L_{\infty}$. Using Barbalat's lemma (Popov (1973)), we conclude that $\lim _{t \rightarrow+\infty}\|\Delta T\|_{2}(., t)=0$.

Note that equations $(21),((9),(17)),(20)$ and (23) correspond to equations $(1),(2),(3)$ and (6) in [Hong (1996). Theorem1], which leads to:

$$
\lim _{t \rightarrow+\infty} \Delta T=0
$$

and thus,

$$
\lim _{t \rightarrow \infty} \Delta \chi_{e}=\lim _{t \rightarrow \infty} \Delta S=0,
$$

with Theorem 1 and the persistent excitation assumption.

\section{SIMULATION RESULTS}

Simulation with computed data is carried out to evaluate the reconstruction performance of the spatially varying input/diffusion adaptive identifier (8),(9). The dissipation parameter $\tau$ is assumed to be known and constant, given by Hoang et al. (1998) empirical model. Simulations are performed using MATLAB/Simulink.

Since the identifier is infinite-dimensional, the bsplinecubic Galerkin method is used in order to implement it (Banks and Kunisch (1989)). The simulated data is generated by using:

$$
\left\{\begin{array}{l}
\chi_{e}(x, t)=\left(0.1+5 x+2 x^{2}+4 x^{3}\right) \mathbf{1}(t) ; \tau=0.05 \\
S(x, t)=\frac{10^{5}}{\sqrt{2 \pi \sigma}} \exp \left(\frac{-(x-\mu)^{2}}{2 \sigma^{2}}\right) \mathbf{1}(t) \\
x \in[0,1], \quad t \in[0,1], \quad d x=0.05, \quad d t=0.01 .
\end{array}\right.
$$

The choice of $\chi_{e}, \tau$ and $S$ is motivated by the example proposed by Zou et al. (2003), where it was assumed that the diffusion coefficient has a monomial monotonically increasing function and the heating source undergoes a spatial Gaussian form. These parameters were considered constant in time. Fig. 1 presents the space variations of $\chi_{e}$ and $S$ used in the mock-up data to generate $T$. 

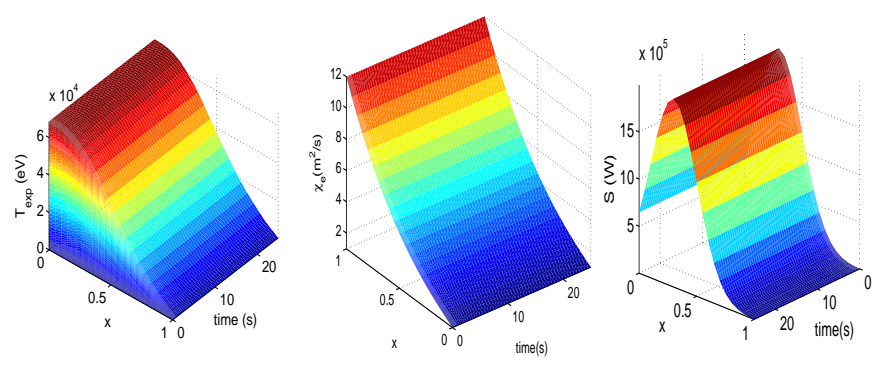

Fig. 1. T, $\chi_{e}$ and $S$ for the mock-up example

To evaluate the reconstruction performance using the adaptive identifier (8),(9), the initialization of the filter was arbitrary. The choice of the bases dimensions in the Galerkin formulation is as follows. For $\chi_{e}$ and $S$, we have chosen a dimension of $n=9$, whereas for $T$, we have chosen $n=20$ for its space basis dimension. It is a good trade-off between precision and convergence rate as shown in Fig. 2. Note that the space basis dimension of $T$ is related to the number of required sensors as we are using the bsplinesGalerkin method. In practice (for the tokamak facility) more sensors are available.
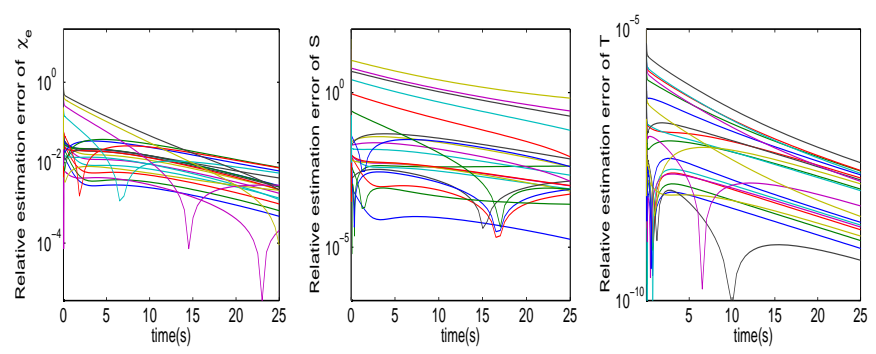

Fig. 2. Estimation errors of $\chi_{e}, S$, and $T$ for the mock-up example

From Fig. 2, the estimation of $\chi_{e}, S$ and $T$ using the adaptive identifier $(8),(9)$ is satisfactory. This figure shows the time evolution of each node relative estimation error. The filter needs few iterations to converge to the original variables. The choice of $\vartheta_{i}, i=0,1,2$ is crucial. From the simulation, we observe that for each $\vartheta_{0}$ corresponds a couple of $\left(\vartheta_{1}, \vartheta_{2}\right)$ and increasing these estimation/adaptation gains leads to faster state and parameters $\left(\chi_{e}\right.$ and $S$ ) convergence. However, similarly to the gradient search method, beyond some points, larger sizes lead to oscillations and even slow convergence.

\section{CONCLUSION}

In this paper we have studied and tested state input and parameter adaptive estimation for a linear parabolic PDE representing the plasma heat transport in a Tokamak. Three related problems were considered. First, only space-varying parameter/input were considered. The proposed identifier tested in simulation gives good results. For distributed slowly time-varying and time-varying input/parameter with known upper bounds, only theoretical results were given. More simulations on computed and real data are needed to establish the performance of the proposed technique.

In comparison with our previous results using a Kalman filter in the finite dimensional framework, the present approach provided a clearer analytical framework but is more sensitive to tuning parameters. In this paper, noise measurement implication is not investigated. In our future works, this question will be addressed.

\section{REFERENCES}

Banks, H.T. and Kunisch, K. (1989). Estimation techniques for distributed parameter systems.

Baumeister, J., Scondo, W., Demetriou, M.A., and Rosen, I.G. (1997). On-line parameter estimation for infinitedimensional dynamical systems. SIAM J. CONTROL OPTIM., 35(02), 678-713.

Demetriou, M.A. and Rosen, I.G. (1997). Robust adaptive estimation for parabolic distributed parameter systems. 3448-3453.

Evans, L.C. (2010). Partial differential equations: Graduate studies in Mathematics, volume 19. American Mathematical Society, 2 edition.

Hinton, F. and Hazeltine, R. (1976). Theory of plasma transport in toroidal confinement systems. Reviews of Modern Physics, 48(2), 239-308.

Hoang, G., Saoutic, B., Guiziou, L., Basiuk, V., Bécoulet, A., Budny, R., Clairet, F., Colas, L., Devynck, P., Gil, C., Joffrin, E., Litaudon, X., Ségui, J.L., Voitsekhovitch, I., and Zou, X. (1998). Magnetic shear effects on confinement and electron heat transport in Tore Supra discharges with electron heating. Nuclear Fusion, 38(1), $117-132$.

Hong, K. (1996). Asymptotic behaviour of a coupled dynamic system: Application to adaptive systems. IFAC 13th Triennial World Congress.

Mechhoud, S., Witrant, E., Dugard, L., and Moreau, D. (2013a). Combined distributed parameters and source estimation in tokamak plasma heat transport. European Control Conference, submitted.

Mechhoud, S., Witrant, E., Dugard, L., and Moreau, D. (2013b). Joint diffusivity and source estimation in tokamak plasma heat transport. American Control Conference.

Orlov, Y. and Bentsman, J. (2000). Adaptive distributed parameter systems identification with enforceable identifiability conditions and reduced-order spatial differentiation. IEEE Transaction On Automatic Control, 45(02), 203-216.

Popov, V.M. (1973). Hyperstability of control systems. Bucuresti: Editura Academiei; Berlin: Springer.

Raue, A., Kreutz, C., Klingmuller, U., and Timmer, J. (2010). Addressing parameter identifiability by modelbased experimentation. IET. Syst. Biol., 5(2), 120-130.

Wada, S., Mitsunaga, K., Suemitsu, H., and Matsu, T. (2006). Adaptive estimation of time-varying parameters and its application to time-derivative tracker. SICEICASE International Joint Conference.

$\mathrm{Yu}, \mathrm{W}$. and Seinfeld, J.H. (1988). Identification of parabolic distributed parameter systems by regularization with differential operators. Journal of mathematical analysis and applications, 132, 365-387.

Zou, X.L., Giruzzi, G., Artaud, J.F., Bouquey, F., Clémençon, A., Darbos, C., Dumont, R.J., Guivarch, C., Lennholm, M., Magne, R., and Ségui, J.L. (2003). Electron heat transport and ECRH modulation experiments in Tore Supra tokamak. Nuclear Fusion, 43, 1411-1420. 